\title{
Current practices in the management of diabetic nephropathy
}

\author{
${ }^{1} \mathrm{G}$ Chan, ${ }^{2} \mathrm{~S} \mathrm{C}-\mathrm{W}$ Tang \\ ${ }^{1}$ Resident; 'Professor, Department of Medicine, University of Hong Kong, Queen Mary Hospital, Hong Kong
}

\begin{abstract}
The social and economic burden of treating patients with diabetes mellitus (DM) is rapidly rising. Current projections estimate the global prevalence of individuals with DM to rise from $6.4 \%$ (285 million) in 2010 to $7.7 \%$ (439 million) in 2030.' The main problem with this disease entity is its propensity to incur macro- and micro-vascular complications over time, including diabetic nephropathy (DN).

Diabetic nephropathy affects approximately one-third of individuals with diabetes. It is the leading cause of end-stage renal disease (ESRD) worldwide, accounting for $42 \%$ of all patients on renal replacement therapy (RRT) in the United States. ${ }^{2}$ The magnitude of this problem has continued to grow in the face of an inexorable rise in the number of diabetic patients. The search for therapeutic modalities to stem this tide remains the quest of many nephrologists. One of the hallmarks of DN is increased urinary protein excretion, and microalbuminuria has long been proposed as an early manifestation of this disease. ${ }^{3,4}$ Albuminuria and chronic kidney disease (CKD) are strong determinants of cardiovascular disease and to a large extent, the survival of patients with DN is determined by cardiovascular morbidity. Although there remains no cure at present, treatment options to prevent or slow disease progression are available. In this update, we aim to address the current armamentarium in the management of DN.
\end{abstract}

KEYWORDS Diabetic nephropathy, clinical trials, proteinuria, RAS blockade, chronic kidney disease, simultaneous pancreas and kidney transplants

DECLARATIONS OF INTERESTS No conflicts of interest declared.

\section{THERAPEUTIC APPROACH TO DIABETIC NEPHROPATHY}

\section{Glycaemic control optimisation}

Damage to the renal microvasculature causes diabetic nephropathy (DN), and correlates well with the levels of glycaemic control in diabetic patients. The UK Prospective Diabetes Study (UKPDS) trial randomised patients with type 2 diabetes mellitus (DM) to receive either intensive glucose lowering or conventional therapy. At a median follow-up of 10 years, which achieved a median glycated haemoglobin ( $\mathrm{HbAlc}$ ) difference of $0.9 \%$ (7.0\% vs $7.9 \%$ ), the risk reduction of incident microalbuminuria in the intensive arm was $33 \% .{ }^{5}$ These findings are in keeping with the results of the Diabetes Control and Complications Trial (DCCT) which recruited patients with type I DM. The current recommendation is to target a $\mathrm{HbAlc}<7 \%$ (the American Diabetes Association standard) in an attempt to balance the risk of hypoglycaemia with the clear benefit of renoprotection in this cohort.

\author{
Correspondence to S Tang \\ Rm 4 I5 Professorial Block \\ Queen Mary Hospital \\ 102 Pokfulam Road \\ Hong Kong
}

tel +852 22553879

e-mail

scwtang@hku.hk
Blood pressure control

Hypertension is prevalent in patients with diabetes, even when clinically evident renal involvement is absent. In $\mathrm{DN}$, blood pressure control, irrespective of the agent used, postpones renal insufficiency, delays its progression, and improves survival. Prospective observational study data from the UKPDS 36 trial revealed that every 10 $\mathrm{mm} \mathrm{Hg}$ reduction in systolic blood pressure is associated with a $12 \%$ reduction in any diabetes-related complication. ${ }^{5}$ This was supported by the more recent post hoc analyses of the Reduction of Endpoints in NIDDM with the Angiotensin II Antagonist Losartan (RENAAL) trial, which found that every $10 \mathrm{mmHg}$ increase in baseline systolic blood pressure was associated with an enhanced risk of end-stage renal disease (ESRD) or a death rate of $6.7 \% .^{6}$ The National Kidney Foundation advises that the blood pressure goals should be less than $130 / 80 \mathrm{~mm} \mathrm{Hg}$ for non-proteinuric patients and $125 / 70 \mathrm{mmHg}$ for those with proteinuria. 


\section{Blockers of the renin-angiotensin system (RAS)}

There is little doubt that renin-angiotensin (RAS) blockade confers protection benefit in patients with DN. Angiotensin-converting enzyme inhibitors (ACEi) and angiotensin II receptor blockers (ARB) are widely used to control blood pressure in patients with diabetes. Moreover, they are superior to other antihypertensives in DN by virtue of their capacity to reduce proteinuria, via reduction of intraglomerular pressure, which is considered to be an independent modifiable risk factor for disease progression. In fact, the landmark trials of Lewis et al., the Irbesartan Diabetic Nephropathy Trial (IDNT), ${ }^{7}$ and RENAAL $^{6}$ demonstrated clearly the renoprotective benefits of both $A C E i$ and $A R B$ to be independent of blood pressure control. In the post-hoc analysis of RENAAL, ${ }^{8}$ reduction in albuminuria in the first six months of therapy with losartan was linearly related to the degree of long-term renoprotection: every $50 \%$ reduction in albuminuria in the first six months was associated with a risk reduction of $45 \%$ for ESRD during later follow-up.

With regard to the comparative effectiveness of $A C E i$ and ARB in DN, there are little data to favour one over the other. The general consensus is that they can be employed interchangeably as required, usually when patients develop intractable cough associated with ACE inhibition. This is supported by DETAIL,' a randomised control trial designed to compare the ACEi enalapril to the ARB telmisartan in 250 patients with type 2 DM complicated by early nephropathy, as defined by albuminuria. At five years, there was no significant difference in decline of glomerular filtration rate (GFR) between the study arms. Both groups were similar with regard to secondary endpoints, including GFR, serum creatinine, quantity of albuminuria, and ESRD.

A combination therapy of ACEi plus ARB has been suggested in an attempt to achieve better RAS blockade. However, no trial to date has unequivocally shown combination therapy to retard progression of CKD in the DM cohort. Moreover, the Ongoing Telmisartan Along and in Combination with Ramipril Global Endpoint Trial (ONTARGET) 10 showed combination therapy to worsen major renal outcomes of dialysis or doubling of serum creatinine as compared with ramipril or telmisartan alone. This trial, which recruited 25,620 subjects at high risk of vascular events, including those with diabeties, casts doubt over the role of dual blockade in DN. Furthermore, the risk of refractory hyperkalaemia, particularly in patients with significant renal impairment, often prevents the use of both agents simultaneously. At this juncture, combination therapy is not recommended in the management of DN.

The reactive increase in plasma renin activity (the capacity of renin to convert angiotensinogen to angiotensin), in association with ACEi/ARB therapy, has been well documented. In theory, direct renin inhibition as a method for RAS down-regulation may be more complete and effective by acting at a higher level within the pharmacological axis. In 2007, the United States Food and Drug Administration approved aliskiren, a direct renin inhibitor, for the treatment of hypertension. The Aliskiren in the Evaluation of Proteinuria in Diabetes (AVOID) trial" enrolled 599 hypertensive patients with DN and found aliskiren, in addition to ARB losartan, to possess anti-proteinuric effects defined by a $20 \%$ reduction in urinary albumin-to-creatinine ratio, independent of blood pressure control. However, there are no long-term data and the recent termination of the Aliskiren Trial in Type 2 Diabetes Using Cardio-Renal Endpoints (ALTITUDE), ${ }^{12}$ a large international randomised controlled trial, highlights the risks of using aliskiren in combination with other RAS inhibitors.

Aldosterone antagonism has also been explored as a therapeutic agent in retarding DN. Trial data appears to suggest a benefit, with a reduction in proteinuria, thereby conferring plausible renoprotection, when used either alone or in addition to ACEi/ARB. The risk of hyperkalaemia in combination ACEi plus ARB therapy is significant. Moreover, hard evidence with convincing data showing a reduction in primary end points such as time to reach ESRD is still lacking and the jury is still out on aldosterone antagonism.

\section{Transplantation}

Ever since the 1970s, kidney transplantation has been a modality of RRT available to patients with diabetic ESRD. However, early attempts resulted in high morbidity and mortality. Currently, modern developments in immunosuppression have significantly improved allograft survival in transplant recipients by reducing rejection rates. Available data indicates a $89 \%$ one-year and $66.7 \%$ five-year allograft survival for recipients of cadaveric grafts and a $95.1 \%$ one-year and $80.2 \%$ five-year allograft survival for recipients of living donor transplants. ${ }^{13}$

Combined kidney-pancreas transplantation also merits discussion. The majority are simultaneous pancreas and kidney transplants (SPK), while a small proportion are performed as sequential pancreas after kidney transplant (PAK). Although not widely available, SPK offers a form of RRT in conjunction with the possibility to restore normoglycaemia. The result is freedom from frequent blood sugar monitoring, insulin injections and hypoglycaemia, which undoubtedly offers an improved quality of life following engraftment. ${ }^{14}$ Initially, SPK was reserved for type I DM patients with ESRD. However, there is accumulating evidence to suggest benefit in selected type 2 DM subjects with comparable survival rates. One-year and ten-year patient survival has been quoted at $95 \%$ and $70 \%$, respectively, and, in comparison, SPK transplantation fares similarly to living donor kidney transplantation. ${ }^{15,16}$ 


\section{INTO THE FUTURE: REGENERATION FOR THE NEXT GENERATION}

Although the optimal therapy for $\mathrm{DN}$ continues to evolve, current available strategies are all aimed at slowing disease progression. However, short of a definitive cure, the social and economic burden of patients with DN reaching ESRD will continue to impact healthcare systems in the future. This provides considerable impetus for novel treatments of DN. Significant progress has been made in our understanding of the pathogenic mechanisms, at a molecular level, of this condition. A few novel therapies have emerged. However, the vast majority have focused upon the attenuation of inflammatory pathways. Perhaps, the most exciting news is the prospect of kidney regeneration. Although still in its infancy, and hampered by many complexities and setbacks, the concept of stem cells, as well as stem cell growth factors, present an opportunity for a brighter future.

\section{REFERENCES}

I Shaw JE, Sicree RA, Zimmet PZ. Global estimates of the prevalence of diabetes for 2010 and 2030. Diabetes Res Clin Pract 2010; 87:414. http://dx.doi.org//0.1016/j.diabres.2009.10.007

2 Akmal M. Hemodialysis in diabetic patients. Am J Kidney Dis 200I; 38 (4 Suppl I):SI95-9. http://dx.doi.org/I0.1053/ajkd.200I.27443

3 Mogensen CE, Christensen CK. Predicting diabetic nephropathy in insulin-dependent patients. N Engl J Med 1984; 3 I I:89-93. http:// dx.doi.org/I0.1056/NEJMI98407| 23 II0204

4 Mogensen CE. Microalbuminuria predicts clinical proteinuria and early mortality in maturity onset diabetes. N Engl J Med 1984 310:356-60. http://dx.doi.org/ I0.1056/NEJMI98402093 I00605

5 Adler Al, Stratton IM, Neil HA et al. Association of systolic blood pressure with macrovascular and microvascular complications of type 2 diabetes (UKPDS 36): prospective observational study. BMJ 2000; 32 I:4 I 2-9. http://dx.doi.org/ I0.I I 36/bmj.32I.7258.4I2

6 Bakris GL, Weir MR, Shanifar S. Effects of blood pressure level on progression of diabetic nephropathy: results from the RENAAL study. Arch Intern Med 2003; 163:I555-65. http://dx.doi. org/10.1001/archinte.163.13.1555

7 Lewis EJ, Hunsicker LG, Bain RP et al. The effect of angiotensinconverting-enzyme inhibition on diabetic nephropathy. The Collaborative Study Group. N Engl J Med 1993; 329:1456-62. http://dx.doi.org/I0.I056/NEJMI993 I I I 3292004

8 de Zeeuw, Remuzzi G, Parving $\mathrm{HH}$ et al. Proteinuria, a target for renoprotection in patients with type 2 diabetic nephropathy: lessons from RENAAL. Kidney Int 2004; 65:2309-20. http://dx.doi. org/I0.IIII/j.I523-I755.2004.00653.x

9 Barnett $\mathrm{AH}$, Bain SC, Bouter $\mathrm{P}$ et al. Angiotensin-receptor blockade versus converting-enzyme inhibition in type 2 diabetes and nephropathy. N Engl J Med 2004; 35I:|952-6I. http://dx.doi. org/10.1056/NEJMoa042274

\section{CONCLUSION}

In summary, the prevalence of DM with resultant nephropathy leading to end-stage renal failure is rising rapidly. Therapeutic strategies have initially focused upon optimisation of blood pressure and glycaemic control. Subsequent realisation of the benefits of RAS blockade has led to the use of different pharmacological agents to manipulate this axis. However, these approaches will only delay the natural progression of DN and many will still reach end-stage renal failure, requiring RRT. As a form of RRT, combined kidney-pancreas transplantation, where it is available, appears to be an attractive option but future developments most likely rely upon the prospect of stem cell transplantation and kidney regeneration.
I0 Mann JF, Schmieder RE, McQueen M et al. Renal outcomes with telmisartan, ramipril, or both, in people at high vascular risk (the ONTARGET study): a multicentre, randomised, double blind, controlled trial.Lancet 2008; 372:547-53. http://dx.doi.org/I0.1016/ SOI40-6736(08)6I236-2

II Parving $\mathrm{HH}$, Persson F, Lewis JB et al. Aliskiren combined with losartan in type 2 diabetes and nephropathy. N Engl J Med 2008; 358:2433-46. http://dx.doi.org// 0.1056/NEJMoa0708379

12 Parving $\mathrm{HH}$, Brenner BM, McMurray JJ et al. Aliskiren trial in type 2 diabetes using cardio-renal endpoints (ALTITUDE): rationale and study design. Nephrol Dial Transplant 2009; 24:1663-7I. http://dx. doi.org/10.1093/ndt/gfn721

13 Port FK, Merion RM, Goodrich NP et al. Recent trends and results for organ donation and transplantation in the United States, 2005. Am JTransplant 2006; 6: 1095-100. http://dx.doi.org/I0.1 I I //j. I6006/43.2006.0I268.x

14 Nathan DM, Fogel H, Norman D et al. Long-term metabolic and quality of life results with pancreatic/renal transplantation in insulin-dependent diabetes mellitus. Transplantation 1991; 52:8591. http://dx.doi.org/10.1097/00007890-199/07000-00018

I5 Rayhill SC, D'Alessandro AM, Odorico JS et al. Simultaneous pancreaskidney transplantation and living related donor renal transplantation in patients with diabetes: is there a difference in survival? Ann Surg 2000; 231:417-23. http://dx.doi.org//0.1097/00000658-20000300000015

I6 Reddy KS, Stablein D, Taranto S et al. Long-term survival following simultaneous kidney-pancreas transplantation versus kidney transplantation alone in patients with type I diabetes mellitus and renal failure. Am J Kidney Dis 2003; 4I:464-70. http://dx.doi. org/I0.1053/ajkd.2003.50057 


\section{SELF-ASSESSMENT QUESTIONS}

I. Which ONE of the following statements is TRUE with regard to management of diabetic nephropathy?

A. Aliskiren in combination with an angiotensin receptor blocker has been validated for the treatment of diabetic nephropathy.

B. Aliskiren in combination with an ACEi has been validated for the treatment of diabetic nephropathy.

C. Aim blood pressure control to be less than 135/85 mm $\mathrm{Hg}$ in proteinuric diabetic nephropathy patients.

D. Tight glycaemic control to achieve $\mathrm{HbAlc}<7.5 \%$ is advocated (the American Diabetes Association standard).

E. Simultaneous pancreas and kidney transplant is a worthy consideration in those who reach end-stage renal disease requiring renal replacement therapy.

2. Which ONE of the following statements is CORRECT with regard to diabetic nephropathy and renal replacement therapy (RRT)?

A. Diabetic control may worsen after the initiation of peritoneal dialysis.

B. Diabetic control will improve after the initiation of haemodialysis.

C. Simultaneous pancreas and kidney (SPK) transplant should only be performed in patients with end-stage renal disease secondary to type I diabetes.

D. The outcomes of simultaneous SPK are worse than living donor renal transplantation.

E. The insulin requirement in diabetic patients approaching RRT is increased.
3. Which ONE of the following statements is TRUE with regard to renin-angiotensin blockade in diabetic nephropathy?

A. Dual blockade carries a risk of hyperkalaemia.

B. Dual blockade is currently validated for the treatment of diabetic nephropathy.

C. It is contraindicated in patients with diabetic nephropathy complicated by unilateral renal artery stenosis.

D. It does not require monitoring of renal function at initiation of ACEi or ARB.

E. The pool of data in diabetic nephropathy is significantly larger for angiotensin-receptor blockers compared with angiotensin-converting enzyme inhibitors.

4. Which ONE of the following statements is TRUE with regard to diabetic nephropathy?

A. Haematuria is an independent modifiable risk factor for progression of diabetic nephropathy.

B. Characteristically presents with bilaterally small kidney sizes on ultrasound.

C. Can be associated with nephrotic range proteinuria.

D. Accounts for $10 \%$ of all patients with end-stage renal disease worldwide.

E. Demonstrates $\sim 95 \%$ concordance with diabetic retinopathy (DMR) such that diabetic patients without diabetic retinopathy are highly unlikely to have diabetic nephropathy.

This paper was originally published as part of the Renal Medicine module in the RCPE Online Continuing Medical Education Programme. Online CME, including the anwers to these questions, is available to Fellows and Members at: http://www.rcpe.ac.uk 